\title{
Fibroqueratoma digital adquirido multirramificado. Caso clínico
}

\author{
Multibranched acquired digital fibrokeratoma. Clinical case \\ María del Mar Torres Infante ${ }^{1}$, Juan Manuel Muriel Sánchez y Pedro Montaño Jiménez² \\ 'Máster Oficial en Nuevas Tendencias Asistenciales en Ciencias de la Salud. Sevilla, España. ${ }^{2}$ Departamento Podología, Facultad Enfermería, \\ Fisioterapia y Podología. Sevilla, España
}

Palabras clave:

Fibroqueratoma, fibroma digital, caso clinico, tumor Koenen.

Keywords:

Fibrokeratoma, digital fibroma, clinical case, Koenen tumor.

\begin{abstract}
Resumen
El fibroqueratoma es una lesión tumoral benigna del aparato ungueal. Se trata de una lesión adquirida que se desarrolla a partir de un collarete epidérmico queratósico que rodea su base. Son tumores cutáneos, de naturaleza benigna, asintomáticos, generalmente de pequeño tamaño, consistencia firme, encapsulados y con una capa superficial gris perlada. Tienen una etiología desconocida. Morfológicamente, aparecen como una neoformación de forma semiesférica recubierta completamente por piel normal sonrosada y, generalmente, son asintomáticos. Inusualmente, los podemos encontrar como lesiones multilobuladas o multirramificadas. El diagnóstico es fundamentalmente clínico, apoyado en el informe de anatomía patológica de la lesión tras su escisión quirúrgica. El presente artículo presenta un caso clínico de escisión quirúrgica de un fibroqueratoma mostrándolo como una buena alternativa de tratamiento que ofrece una solución definitiva, sencilla y sin complicaciones para el paciente.
\end{abstract}

\begin{abstract}
Fibrokeratoma is a benign tumor of the nail. It is an acquired injury that develops from a keratotic epidermal collar that surrounds its base. They are benign, asymptomatic skin tumors, generally small in size, firm in consistency, encapsulated and with a pearly gray surface layer. They have an unknown etiology. Morphologically, they appear as a hemispherical neoformation completely covered by normal skin and are generally asymptomatic. Unusually, we can find them as multi-lobed or multi-branched lesions. Its diagnosis is mainly clinical, supported by the pathological report of the lesion after its surgical excision. The present paper presents a clinical case of a surgical excision of a fibrokeratoma showing it as a good treatment alternative that offers a definitive, simple and uncomplicated solution for the patient.
\end{abstract}

\section{INTRODUCCIÓN}

El fibroqueratoma es una lesión tumoral benigna del aparato ungueal, descrito por primera vez por Bart y cols. en 1968'. Se trata de una lesión adquirida que se desarrolla a partir de un collarete epidérmico queratósico que rodea su base. Su forma de presentación más común es en forma de lesiones solitarias a nivel de las articulaciones interfalángicas distales de manos y pies. Tienen un aspecto rosado, carnoso y asintomático, aunque si aparecen en zonas de presión, roce o fricción pueden llegar a ser dolorosos ${ }^{2}$. Inusualmente, los podemos encontrar como lesiones multilobuladas o multirramificadas $^{3}$.

Los fibroqueratomas son lesiones tumorales englobadas dentro de los fibromas duros. Se caracterizan por ser tumores cutáneos de naturaleza benigna, asintomáticos, generalmente de pequeño tamaño, consistencia firme, encapsulados y con una capa superficial gris perlada ${ }^{1-4}$. Son lesiones relativamente frecuentes. Su localización principal es la articulación interfalángica distal de los dedos de las manos, pudiendo 
aparecer en otras localizaciones anatómicas como la palma o dorso de las manos, dedos del pie, muñeca, rodillas, talones $\mathrm{o}$, incluso, cara anterior de la pierna ${ }^{1,4,5}$.

Este tipo de lesiones suelen aparecer a partir de la segunda década de la vida, especialmente en individuos de entre 30-60 años, siendo más frecuente en el sexo masculino ${ }^{1,2}$. Según Castillo y cols., tienen una incidencia del $1.3 \%$, después de revisar más de 20.000 casos en un estudio descriptivo y retrospecti$v^{6}{ }^{6}$. Por otro lado, Fragoso y cols. obtuvieron una incidencia de $5.2 \%$ de tumores fibrosos, sin hacer distinción alguna ${ }^{7}$.

Son lesiones de etiología desconocida. Muchos autores lo relacionan con pequeños traumatismos, pero no existe evidencia científica suficiente acerca de esta relación $n^{2,4,8}$. Suelen ser lesiones de aparición repentina y de velocidad de crecimiento variable. Pueden llegar a alcanzar hasta un centímetro de tamaño ${ }^{9}$.

Morfológicamente, aparece como una neoformación de forma semiesférica recubierta completamente por piel normal sonrosada, en ausencia de dermatoglifos. En ocasiones, se puede observar una hiperqueratosis puntiaguda apical. Surge de un collarete epidérmico queratósico descamativo que en sus inicios pasa desapercibido, llegando a alcanzar hasta los dos centímetros de tamaño, aunque generalmente no suele superar los 1.5 centímetros ${ }^{2,4,8,9}$. En cuanto a su clínica, se presenta como una lesión asintomática, aunque puede causar dolor cuando se traumatiza, generalmente por el roce del zapato. Su forma de presentación más común es tipo pápula, sésil o pediculada con una coloración similar a la piel que la rodea ${ }^{1,9}$.

El diagnóstico diferencial del fibroqueratoma digital adquirido incluye las siguientes lesiones: liquen simple crónico, tumores de Koenen, quistes mixoides digitales, verruga vulgar, fibroma de vaina tendinosa, cuerno cutáneo, poroma ecrino y queloides ${ }^{1,2,4,8,9}$. Cabe destacar que los tumores de Koenen son considerados en la literatura científica un tipo de fibroqueratoma que ocurre de forma múltiple en pacientes con esclerosis tuberosa ${ }^{3}$.

\section{CASO CLÍNICO}

El caso clínico que se presenta corresponde a una mujer de 49 años, sin antecedentes de interés que acude al Área Clínica de Podología de la Universidad de Sevilla por presentar una lesión multirramificada de tejido fibroso y de consistencia dura en la uña del primer dedo del pie derecho de varios años de evolución (Figura 1).

En la exploración se observa que la tumoración está dañando la lámina ungueal debido a que presenta un surco inferior a lo largo de toda su longitud. La paciente indica que ha eliminado varias veces la lesión, pero sin éxito puesto que vuelve a salir y refiere que ha notado un aumento de tamaño a lo largo del tiempo.

En este caso, el diagnóstico fue fundamentalmente clínico, basado en las características morfológicas, su localización, periodicidad y ausencia de sintomatología, el cual se confirmó posteriormente con el informe de anatomía patológica tras realizar una escisión/biopsia o biopsia de tipo escisional.

La paciente dio su consentimiento para someterse a la intervención quirúrgica, en la cual se incluía la realización de fotografías clínicas y su posterior divulgación con fines científicos.

Se describe a continuación el procedimiento quirúrgico realizado. Tras anestesia troncular del primer dedo con mepivacaína al $2 \%$, se procedió con una incisión longitudinal en eponiquio de 3-4 de milímetros aproximadamente con una hoja de bisturí del número 15 (Figura 2).

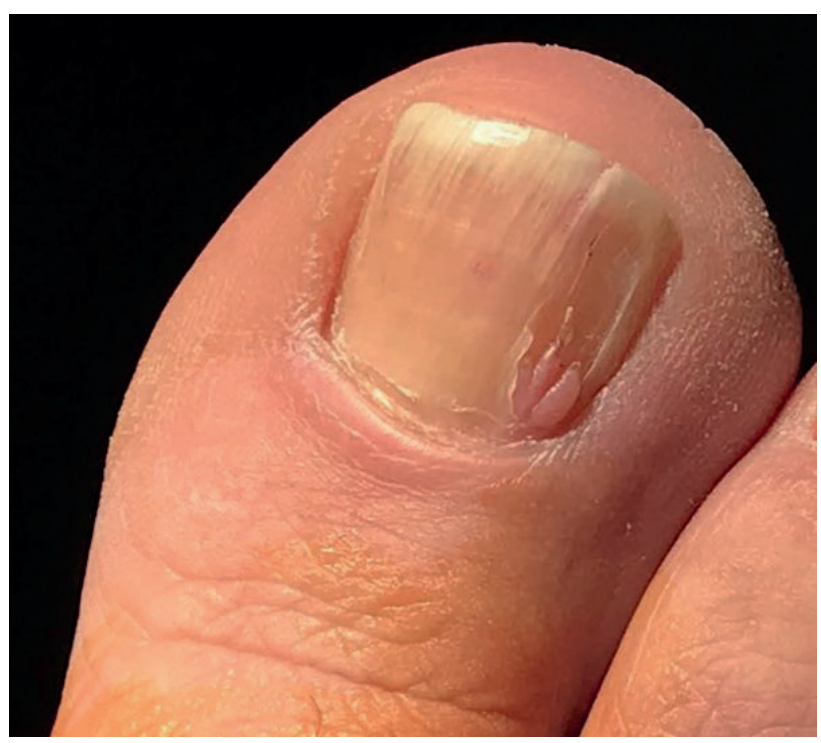

Figura 1. Fibroqueratoma digital adquirido.

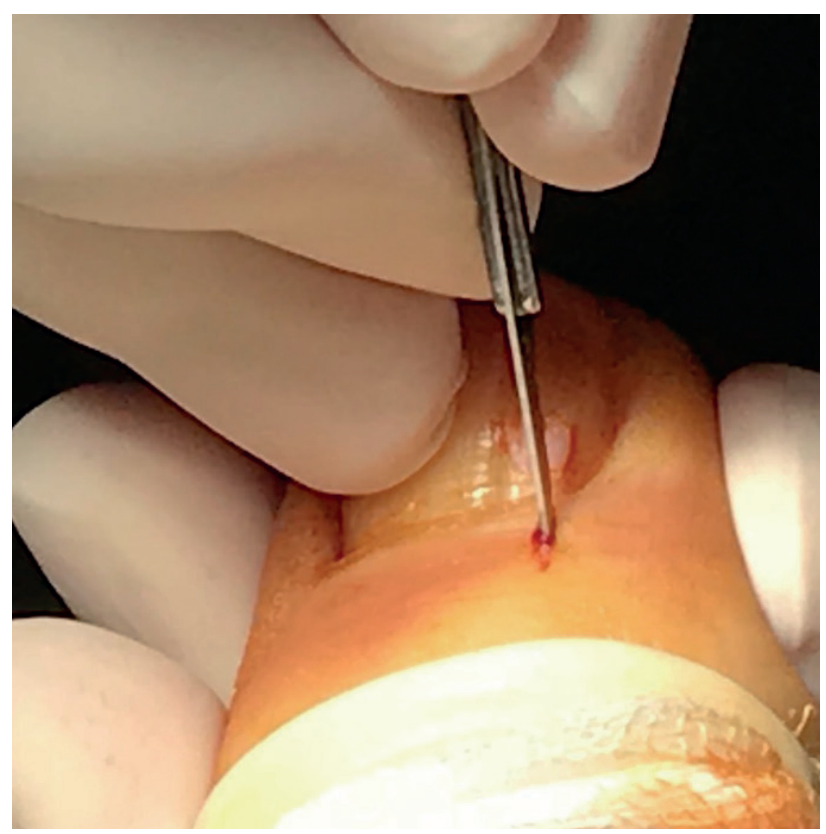

Figura 2. Incisión longitudinal de eponiquio. 
Con ayuda de unas pinzas de tipo Adson y bisturí, se realizó una separación meticulosa de la lesión y de la piel sana del eponiquio con la precaución de no dañar la piel que lo cubre dorsalmente y con la finalidad de exponer dicha lesión en su totalidad (Figura 3). Una vez que la lesión estuvo totalmente expuesta se procedió con un resecado amplio de la misma poniendo especial cuidado en eliminarla desde la base y con la cautela de no dañar tejidos contiguos (Figura 4); finalizado el procedimiento se realizó un lavado con suero fisiológico para eliminar posibles restos. Se efectuó una aproximación de los bordes para ver el resultado final y se procedió a la sutura de la incisión con monofilamento no absorbible 4/0 (Figura 5); al finalizar se aplicó un apósito absorbente no adherente impregnado en povidona yodada, gasas y vendaje semicompresivo.

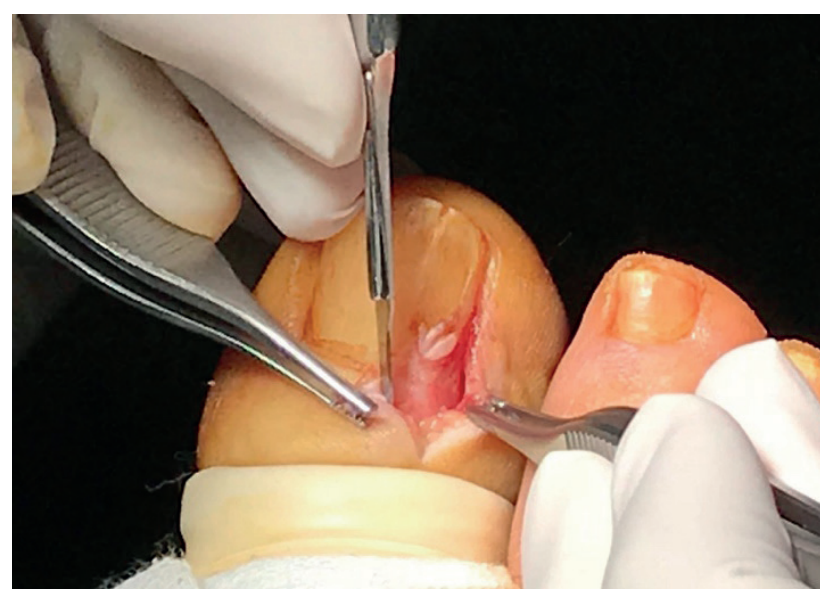

Figura 3. Exposición y localización de la lesión en su totalidad.

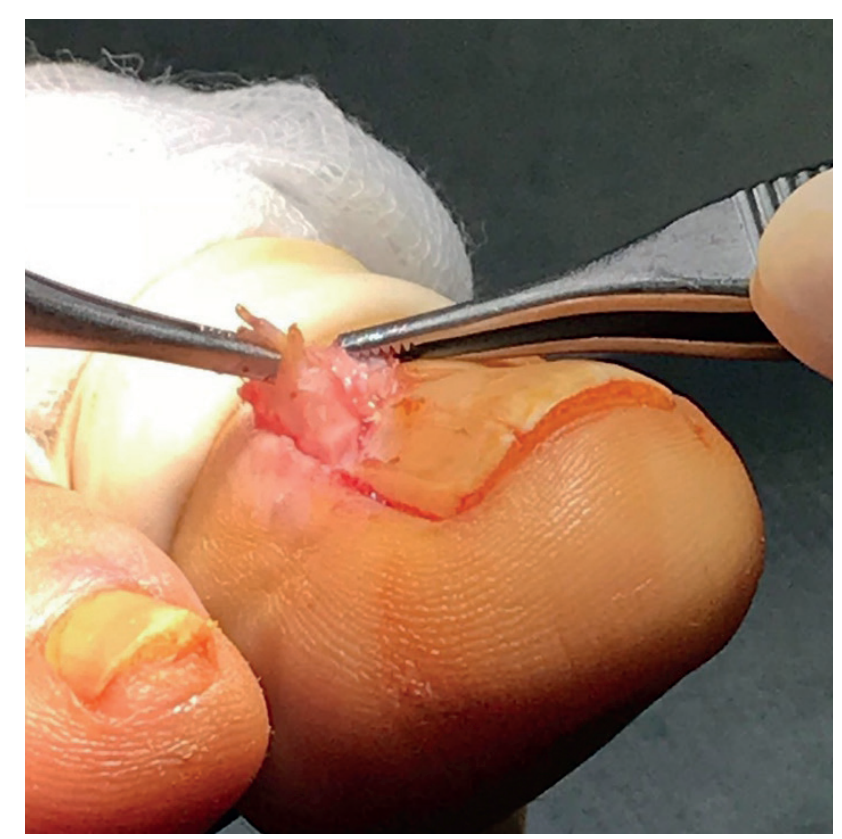

Figura 4. Resecado del tumor desde la base.
Para finalizar se efectuó la medición de la lesión y se envió al servicio de anatomía patológica (Figura 6). Puesto que la lámina ungueal se encuentra sana y no presenta distrofia, se recomendó una escisión de la lesión con la finalidad de preservar la uña intacta.

El resultado de análisis de anatomía patológica fue el siguiente: "Descripción microscópica: epidermis con leve papilomatosis e hiperqueratosis que presenta en dermis un tejido conectivo compuesto por fibras de colágeno en vasos dilatados. Diagnóstico: compatible con fibroqueratoma digital. No hay evidencia de malignidad".

Una vez finalizado el procedimiento quirúrgico, se le indicaron a la paciente pautas generales, las cuales incluyeron reposo relativo tras la intervención, uso de calzado abierto,

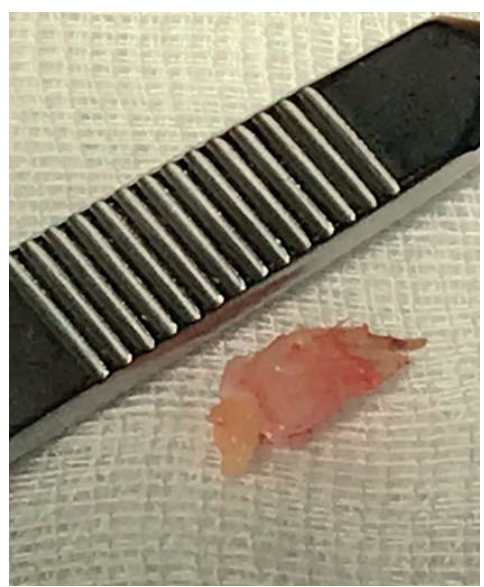

Figura 5. Medición del tumor. $1.82 \mathrm{~cm} \times 0.85$.

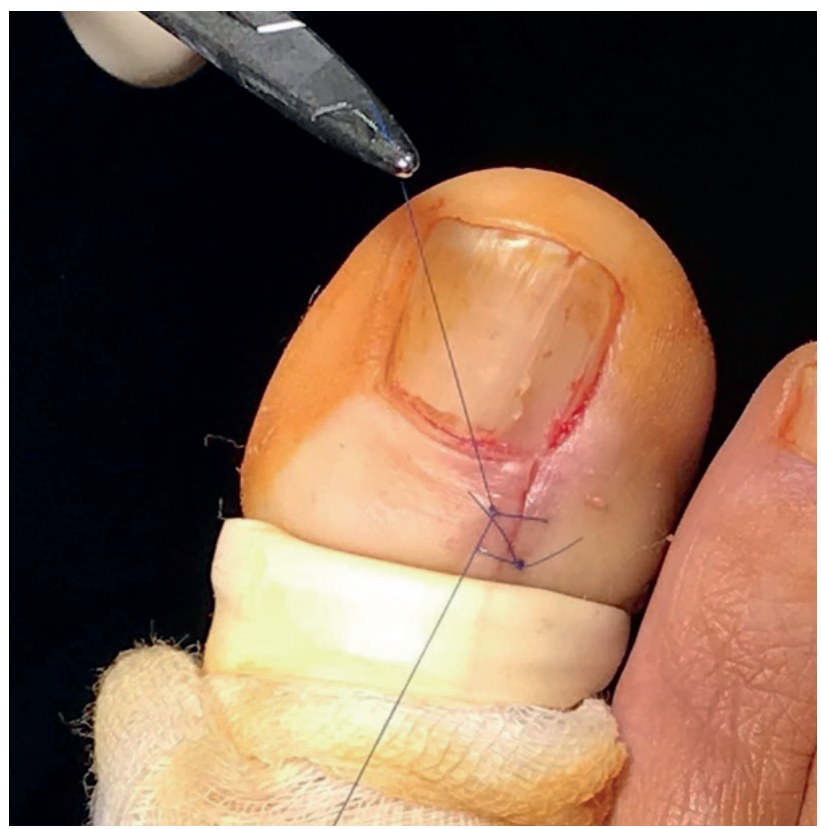

Figura 6. Sutura de la incisión. 


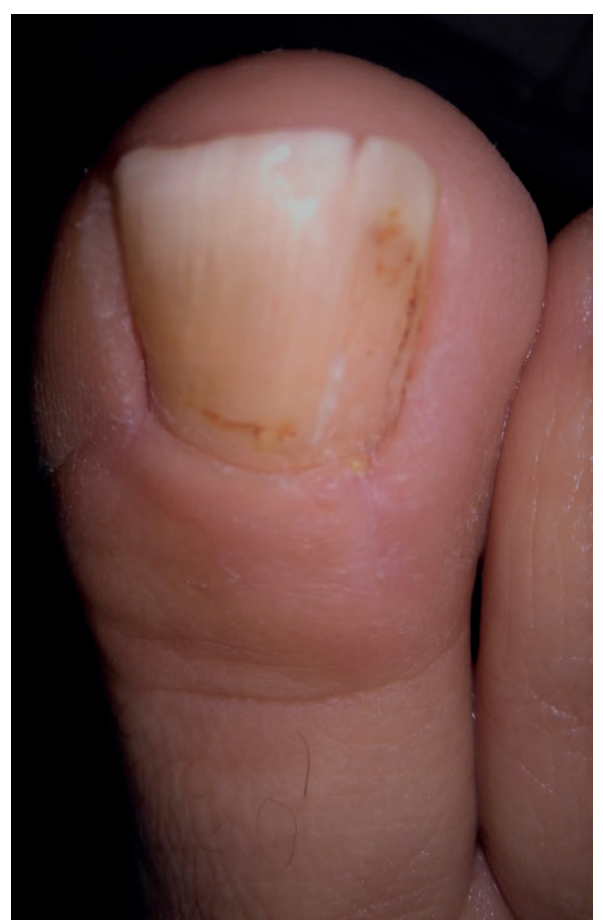

Figura 7. Revisión a los 6 meses.

evitar exposiciones a fuentes de calor y no mojar, además de la prescripción de analgésicos. A los cinco días tras la intervención se realizó la primera cura para la cual se utilizó povidona yodada. Se observó evolución favorable según lo previsto, puesto que no presentaba signos de infección ni inflamación. A los 7 días tras la primera revisión se procedió a la segunda cura, efectuándose el mismo procedimiento que en la anterior y a los 10 días se retiraron los puntos de sutura; se objetivó buena aproximación de los bordes y cierre correcto de la incisión. En la revisión realizada a los 6 meses tras la intervención, no se observaron signos de recidiva de esta, la paciente no presentaba dolor ni cicatriz hipertrófica y la uña no se había visto afectada por la lesión (Figura 7).

La paciente refirió una satisfacción completa con el procedimiento quirúrgico y su resultado estético. Tras varias avulsiones traumáticas de la lesión y su reproducción posterior, refiere haber encontrado una solución definitiva a su problema respetando el crecimiento fisiológico y la morfología de su uña.

\section{DISCUSIÓN}

El fibroqueratoma digital adquirido (ADFK, del inglés "Acquired Digital Fibrokeratoma"), a menudo se diagnostica erróneamente por su parecido con otros tumores benignos y malignos que lo imitan clínicamente; debido a esto, es importante que se realice un diagnóstico y tratamiento oportunos, los exámenes clínicos, dermatoscópicos, histológicos e inmu- nohistoquímicos son métodos útiles para distinguir ADFK de otra entidad similar, confirmándose con una biopsia por escisión ${ }^{10}$.

Si bien se sabe que ADFK no forma parte de ningún síndrome existente, sí que puede estar asociado con infecciones o medicamentos ${ }^{10-13}$. En base a la literatura científica, se han descrito casos de ADFK asociado con infección en pacientes con enfermedad de Hansen ${ }^{14}$. De manera similar, se informó de otro caso de fibroqueratoma multirramificado en un paciente con hipoestesia y hemiplejia secundaria a infarto cerebral $^{10,14}$.

Qiao y cols. reportaron un caso de ADFK asociado con el uso de ciclosporina en un paciente con trasplante renal. El ADFK asociado con ciclosporina puede compartir una patogénesis similar con la del sobrecrecimiento gingival, ya que ambas complicaciones implican una acumulación anormal de colágeno, aunque el mecanismo exacto sigue siendo desconocido $^{10,15}$.

Se pueden usar varias técnicas quirúrgicas para resecar estos tumores, dependiendo de si surgen desde arriba o debajo de la lámina. Las lesiones que aparecen debajo de la lámina requieren la extracción parcial o total de la uña; no obstante, las lesiones que surgen por encima pueden eliminarse mediante escisión seguido de fenolización, o bien vaporización con láser de dióxido de carbono o, más generalmente, resección quirúrgica después de levantar el pliegue ungueal proximal como un colgajo.

En la resección quirúrgica se puede realizar mediante dos incisiones oblicuas en el pliegue ungueal proximal, una en cada extremo del eponiquio; todo el pliegue ungueal proximal se puede levantar para exponer la lesión permitiendo evaluar completamente la base de esta y realizar una escisión completa, evitando una futura regresión, sin dañar la matriz ungueal ${ }^{16}$.

Con respecto a las otras alternativas de tratamiento, el principal inconveniente del procedimiento con láser de dióxido de carbono para los tumores cutáneos radica en la imposibilidad de realizar estudios histopatológicos diagnósticos y una evaluación clara de los márgenes debido a la destrucción completa de la lesión. Esta limitación reduce las indicaciones a tumores en los que el diagnóstico clínico, la biología y la profundidad de las lesiones se puede establecer claramente, y hasta cuándo el tratamiento puede ser realizado por cirujanos con la experiencia adecuada para eliminar las lesiones mediante vaporización con láser de dióxido de carbono ${ }^{17}$.

En base a las principales desventajas del uso del fenol, como el daño químico que se puede producir a la piel sana circundante y tiempos de curación prolongados ${ }^{3}$, los autores de este caso se decantaron por la escisión simple debido a que el fibroqueratoma digital adquirido es una tumoración benigna de la unidad ungueal que no se relaciona histológicamente con las estructuras adyacentes. Ello se traduce en que la disección cuidadosa de la lesión garantiza el crecimiento fisiológico de la placa ungueal sin ninguna secuela. En consonancia con este planteamiento, otros autores han sugerido la 
escisión quirúrgica con colgajo para lesiones periungueales, debido a un menor riesgo de recurrencia y mejores resultados cosméticos ${ }^{10,17,18}$. Aunque el ADFK no muestra regresión espontánea, la escisión es una técnica efectiva y la recurrencia es rara ${ }^{10,17,19,20}$.

En conclusión, el fibroqueratoma digital adquirido es una entidad de diagnóstico complejo por su similitud con otras lesiones dérmicas que, aunque no suele estar asociado a otras patologías, se han descrito diversos casos en la literatura con los que podría estar relacionado. La escisión quirúrgica de un fibroqueratoma multirramificado es una buena alternativa de tratamiento que ofrece una solución definitiva, sencilla y sin complicaciones para el paciente que consigue buenos resultados estéticos y morfológicos que no alteran el crecimiento de la lámina ungueal siempre que se haga mediante un tratamiento adecuado de los tejidos.

\section{CONFLICTO DE INTERESES}

Los autores declaran no tener conflictos de intereses.

\section{FINANCIACIÓN}

Para la realización del artículo no se obtuvo ninguna fuente de financiación externa.

\section{BIBLIOGRAFÍA}

1. Bart RS, Andrade R, KopfAW, Leider M. Acquired digital fibrokeratomas. Arch Dermatol. 1968;97(2):120-9

2. López López D, López López L, Barriuso Cao M, Álvarez-Calderón Iglesias O, Fornos Vieitez B. Fibroqueratoma periungueal adquirido: tratamiento quirúrgico. Rev Int Ciencias Podol. 2007;1(2):47-53.

3. Martínez Nova A, Córdoba Fernández A, Juárez Jiménez J, Rayo Rosado R. Atlas de cirugía ungueal. 2.a ed. Madrid: Médica Panamericana; 2014. p. 255.

4. Izquierdo Cases J. Podología quirúrgica. Madrid: Elsevier; 2006.

5. Betanzos A, Castro I, Salinas C. Fibroqueratoma digital adquirido. Dermatol Pediatr Lat. 2007;5(1):57-9.
6. Castillo D, Zerpa O, Loyo N, López C, Oliver M. Histopatología del cuerno cutáneo: Estudio Retrospectivo de 77 Casos. Derm Venez. 2002;40(3):65-9.

7. Fragoso Gutiérrez A, Medina Bojórquez A, Gutiérrez Vidrio R, Novales Santa Coloma J, Navarrete Franco G, Ramos Garibay A, et al. Tumores cutáneos benignos: correlación clínico-patológica en el servicio de dermatooncología del Centro Dermatológico Pascua de 1995 al 2004. Dermatología Rev Mex. 2006;50(1):9-19.

8. Córdoba Fernández A, Lafuente Sotillos G, Juárez Jiménez J. Fibroqueratoma digital adquirido. Rev Esp Podol. 1998;9(8):415-21.

9. Vázquez Viudas R, Balaciart Gadea A. Fibroqueratoma digital. Caso clínico. Rev Esp Podol. 2012;22(2):76-8.

10. Shih S, Khachemoune A. Acquired digital fibrokeratoma: review of its clinical and dermoscopic features and differential diagnosis. Int J Dermatol. 2019;58(2):151-8. DOI: 10.1111/ijd.14046.

11. Kint A, Baran R, Keyser H De. Acquired (digital) fibrokeratoma. Clin Dermatology. 1984;12(1):816-21.

12. Amarouch $\mathrm{H}$, Aitourghoui M, Ramli I, Zaouri H, Senouci K, Hassam B. Un fibrokératome digital acquis post-staphylococcique: Une nouvelle observation. Press Medicale. 2015;44(7-8):843-5.

13. Pérez-Novo CA, Waeytens A, Claeys C, Cauwenberge P Van, Bachert C. Staphylococcus aureus Enterotoxin B Regulates Prostaglandin E 2 Synthesis, Growth, and Migration in Nasal Tissue Fibroblasts. J Infect Dis. 2008;197(7):1036-43. DOI: 10.1086/528989.

14. Patki $\mathrm{AH}$, Mehta JM. Acquired digital fibrokeratoma in a patient with leprosy. Indian J Lepr. 1992;64(2):205-8.

15. Qiao J, Liu YH, Fang K. Acquired digital fibrokeratoma associated with ciclosporin treatment. Clin Exp Dermatol. 2009;34(2):257-9. DOI: 10.1111/j.1365-2230.2008.02808.x.

16. Moriue T, Yoneda K, Moriue J, Nakai K, Kubota Y. Multibranched acquired periungual fibrokeratoma. JAMA Dermatol. 2014;150(4):4567. DOI: 10.1001/jamadermatol.2013.6631.

17. Yelamos O, Alegre M, Garces JR, Puig L. Periungual acral fibrokeratoma: surgical excision using a banner flap. Actas Dermosifiliogr. 2013;104(9):830-2. DOI: 10.1016/j.ad.2012.10.001

18. Del Pozo J, García Silva J, Peña-Penabad C, Fonseca E. Multiple apocrine hidrocystomas: treatment with carbon dioxide laser vaporization. J Derm Treatment. 2001;12(2):97-100. DOI: 10.1080/09546630131 7085381

19. Shelley WB, Phillips E. Recurring accessory "fingernail": periungual fibrokeratoma. Cutis. 1985;35(5):451-4.

20. Lee $\mathrm{CY}$, Lee KY, Kim KH, Kim YH. Total excision of acquired periungual fibrokeratoma using bilateral proximal nail fold oblique incision for preserving nail matrix. Dermatol Surg. 2010;36(1):139-41. DOI: 10.1111/j.1524-4725.2009.01368.x. 